\title{
A research on the reliability of light-emitting diode based on analyzing of chip image
}

\author{
Jian Ruan and Ke-Yuan Qian \\ Key Laboratory of Information Science and Technology, Graduate School of Tsinghua University at Shenzhen, Shenzhen 518055, China
}

\begin{abstract}
This paper proposes a new method for studying the reliability of high-power light-emitting diode (LED) by analyzing chip images taken from a batch of LEDs which are selected to conduct the accelerated aging test lasting for 1400 hours. To exclude the disturbance of electrode in these images, an image processing algorithm based on projection is used to extract the interested section. An index called "dark point" which is related to non-radiative combination to describe the reliability of LED is proposed. The method is based on analyzing the variation trends of dark points in the extracted section of these images. The results show that the proportion of the dark point increases with aging time increasing. The lifetime of LED with higher increasing rate is shorter. Based on the increasing rate of dark point, the lifetime of the chip can be predicted in an easier way.
\end{abstract}

\section{Introduction}

Compared to traditional incandescent lamps, GaN-based light-emitting diode (LED) has lots of obvious advantages, such as their high efficiency, long lifetime, fast modulation speed, and their linear behaviour under pulsedwidth modulation dimming [1]. Therefore it is believed that LED will replace the widely used incandescent lamps. However, the reliability of LED is still a shortcoming, and the reliability of the high-power LED chip has significant influence on expansion of solid state lighting applications. Therefore the research of the reliability of LED becomes a hot direction.

So far most of research focus on the property of the electrical characteristics and optical characteristics of LED by measuring current-voltage(I-V), capacitancevoltage, output power versus input current(L-I), electroluminescence(EL) and so on. However, these researches still emphasize on measuring the discrete curve of the LED's characteristic curves [2]. They don't essentially reveal the inner mechanisms of the aging of LED. Although there are some researches pointing out that continuous increasement of non-radiative combination centres are the main reason lead to the aging of LED [3-6], they don't give a concrete relation between the density of non-radiative combination centres and aging time. In this paper, a model is used to describe the variation of non-radiative combination centres with aging time.

Also, some researches try to find the relationship between microstructure and luminescence efficiency [79]. And they study the point defects, extended structural defects and other defects on the surface of the chip by atomic force microscopy (AFM), cathode luminescence measurements and transmission electron microscopy to observe the variation of pits on a microchip. However, these methods are complex, and they can only observe the micro area of the chip. In this paper, the structure of the chip in a macro perspective will be studied. The correlation between the dark area that led by the threading dislocations and the non-radiative combination centres will be directly characterized. This method is not only simple, but also very useful. The increasing rate can be used to describe the aging rate of LED. And this index can help us to examine the reliability of LED in a more simple way.

\section{Experiment}

\subsection{Measuring instruments}

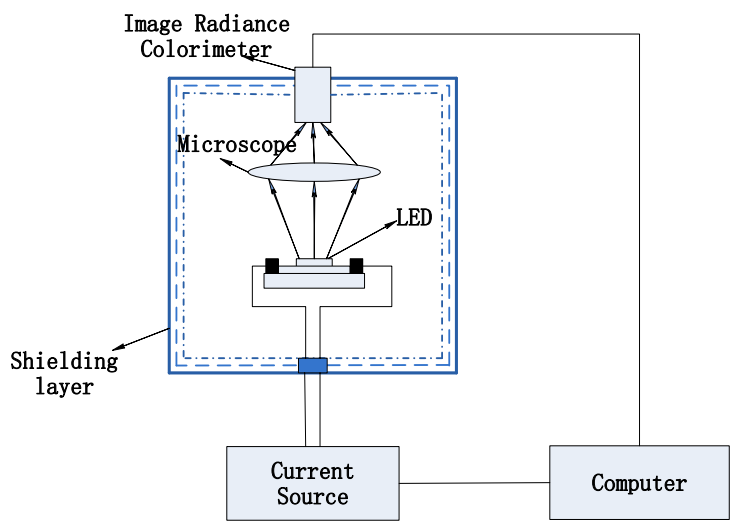

Figure 1. Experimental apparatus for taking images of LED chip. 
Figure 1 shows the experimental apparatus of getting the image of the LED chip. To prevent the chip images from being saturated, the injection current should be as week as $\mu \mathrm{A}$. The current supply source can provide current for the LED whose range is from $10^{-7} \mathrm{~A}$ to $10^{-3} \mathrm{~A}$. The LED works at $30 \mu \mathrm{A}$ in this experiment. A shielding layer is used to shield the ambient light. The LED is put on the stage that can rotate $180^{\circ}$ about the shaft in steps of $0.5^{\circ}$. Through this system, the chip image is taken and imported into the computer, then it can be analyzed.

\subsection{Aging test}

A group of commercial blue LEDs are selected to conduct aging test. The parameters of these samples are shown in Table 1.

Table 1. Parameters of samples.

\begin{tabular}{cc} 
Parameters & Value \\
\hline Power rating & $1 \mathrm{~W}$ \\
\hline Surface Size & $1 \mathrm{~mm} \times 1 \mathrm{~mm}$ \\
\hline Active Layer Thickness & $100 \mathrm{~nm}$ \\
\hline Substrate Temperature & $60^{\circ} \mathrm{C}$ \\
\hline Aging Current & $500 \mathrm{~mA}$ \\
\hline Aging Time & $400 \mathrm{~h}, 700 \mathrm{~h}, 1000 \mathrm{~h}, 1400 \mathrm{~h}$ \\
\hline Samples Amount & 5 \\
\hline
\end{tabular}

\subsection{Data process}

This aim of our study was to analyze the reliability of LED through the variation trend of the images taken from a batch of chips. However, with the LED chip surface covered with complex structures of the electrode, it is hard to find the rule that needed. To exclude the interference of electrode, it is necessary to get rid of the part of electrode in the picture by image processing techniques. Also, these images that got are in large number. It is necessary to use some techniques to deal with these images by computer automagically.

\subsubsection{Image tilt correction}

It is necessary to rotate the image to an appropriate angle before it is segmented. The algorithm of projection is used to get the skew angle. The original image is set in the coordinate system. The method is based on the principle that the active length that the number of pixel value projected on $\mathrm{X}$-axis is the longer when the image is not upright. Just like the Figure 2 (a) and Figure 2(b), the upright image that projected on $\mathrm{X}$-axis is a length of $2 \mathrm{a}$ while another is longer than 2a. According to the geometric features, the oblique image can be easily rotated to an appropriate angle. The result of processed image as Figure 2 (c):

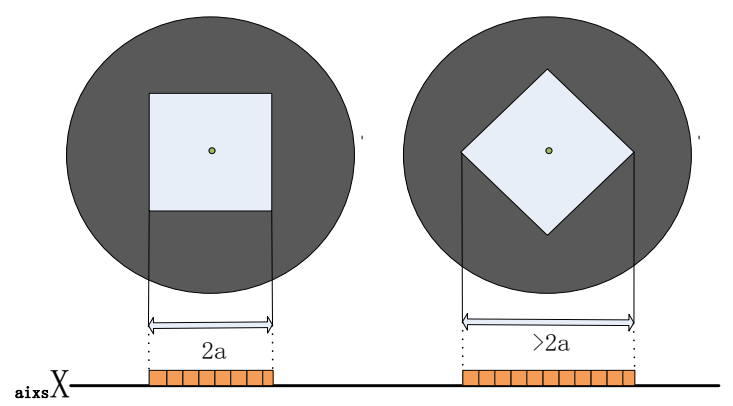

(a)

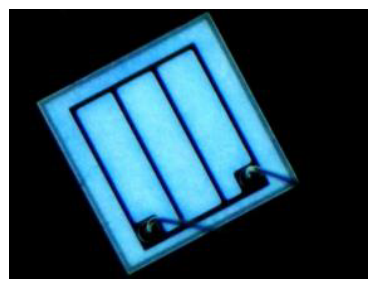

(b)

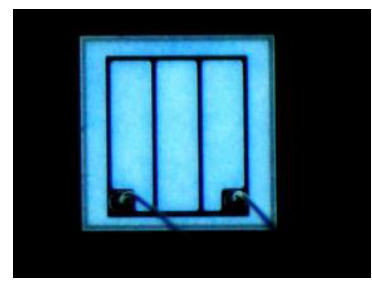

(c)
Figure 2. (a)Sketch map of rotating (b) Original image (c) Image after rotating.

\subsubsection{Segmentation of image}

It is need to identify the electrode that is linear shape. It is easy to find the center of the image by the value that projected in $\mathrm{X}$-axis and $\mathrm{Y}$-axis first. Then the boundary of the image can be found by drifting the center point. The Figure 3 (a) shows the procedure of the algorithm. And the information of the structure can be got by other measurement method.Then the images can be segmented by the information of the chip structure and the position of the boundary. Figure 3 (b) is the image got from the procedure of image rotating. At last the image processed is like Figure 3 (c), the part segmented is signed by red line.

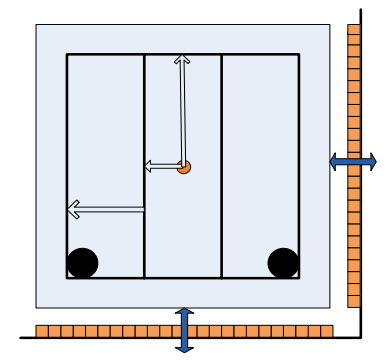

(a)

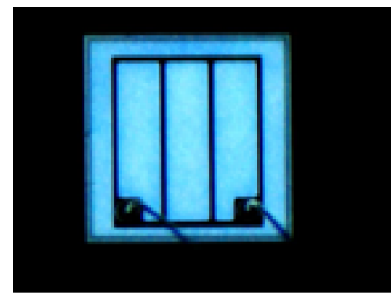

(b)

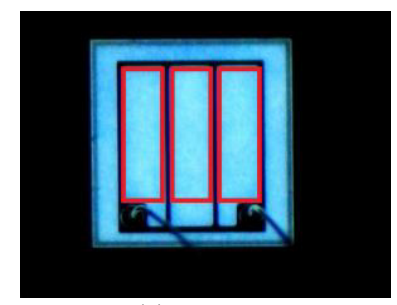

(c)
Figure 3. (a)Sketched image (b) Image before segmenting (c) Image after segmenting. 


\section{Theory}

It is confirmed that non-radiative recombination centers are produced by the ionization of compound of $\mathrm{Mg}-\mathrm{H}_{2}$ [10]. When the energy of carriers in the chip is larger than the chemical energy of $\mathrm{Mg}-\mathrm{H}_{2}$, the compound of $\mathrm{Mg}-\mathrm{H}_{2}$ can be ionized and become defect points. Assume the density of the carriers that can be ionized is $D_{h}$, the initial density of the non-radiative combination center is $D_{o}, D_{c}$ is the density of the defect, and then it will be:

$$
D_{c} \propto D_{0}+\int_{0}^{t} D_{h}(\mathrm{t}) d t
$$

Due to Fermi distribution function, then integrating on the amount of carriers, the ionization energy of $\mathrm{Mg}-\mathrm{H}_{2}$ complexes is $E_{c}$, the Fermi energy is $E_{f}$, the $D_{g}$ is effective density of states on the basics of solid state physics and then integrate on it, there will be:

$$
D_{h}=D_{g} \exp \left(-\frac{E_{c}-E_{f}}{k T}\right)
$$

Due to the directional kinetic energy of carriers in the chip, the energy of carrier must be changed to $E_{c}-E, E$ is the electron kinetic energy, then:

$$
D_{h}=D_{g} \exp \left\{\left[\mathrm{E}_{c}-\mathrm{E}_{f}-\frac{1}{2} m\left(\frac{I}{n q s}\right)^{2}\right] /(\mathrm{kT})\right\}
$$

Then, as $D_{h}$ is an invariant constant, the density of the non-radiative combination centers can be:

$$
D_{c}=D_{0}+D_{h} t
$$

Under the condition of constant current, define a short time process $\Delta \mathrm{t}$, suppose during $\Delta \mathrm{t}$ the carriers needed to transport is $\mathrm{n}$, and suppose the active length is $\mathrm{L}$, under the effect of non-radiative combinations centers with a density of $D_{c}$, the loss of carriers when passing through a length of $\mathrm{dL}$ is :

$$
d n \propto-n D_{c} \times d L
$$

And the formulation can be expressed as:

$$
\int_{n 1}^{n 2} \frac{d n}{n} \propto \int_{0}^{L} D c \times d L
$$

Then it can be expressed as:

$$
n_{1}-n_{0} \propto-\exp \left(-D_{c} L\right)
$$

During a short period $\Delta \mathrm{t}$, the amount of carriers compounded is $\Delta \mathrm{n}$, and $\Delta \mathrm{n} \propto \exp \left(-\mathrm{D}_{\mathrm{c}} \mathrm{L}\right)$. Under constant current I, during a unit time, the carriers compounded should be proportional to I, then it will be:

$$
I_{0} \propto I \exp (-D c L)
$$

Then the efficiency of light output will be:

$$
\eta=\frac{I_{0}}{I}=a \exp (-D c L)
$$

\section{Result and Discussion}

\subsection{Definition of dark points}

It is widely recognized that high density of dislocations, which act as non-radiative recombination centers, are introduced in epitaxial layers grown by using of the heteroepitaxial [11]. And it has been proved that the failure of an LED is closely related to the increasing of non-radiative recombination centers during its lifetime. With aging time increasing, the non-radiative recombination defects increases in the active region of the LED. G.Meneghesso found [7-9], when the defects of LED aggregated to an extent, it will change the surface topography of the chip. Under the atomic force microscopy, it can be observed that the dark areas increase in the surface of the chip after aging. If the images of the chip are taken, it can be found some areas in the images that the pixels value is low which can be seem equivalent to dark areas. Then it can be observed the dark points by counting dark pixels in these images. Before the definition of dark point, Figure 4 is given to describe it in an intuitive way.

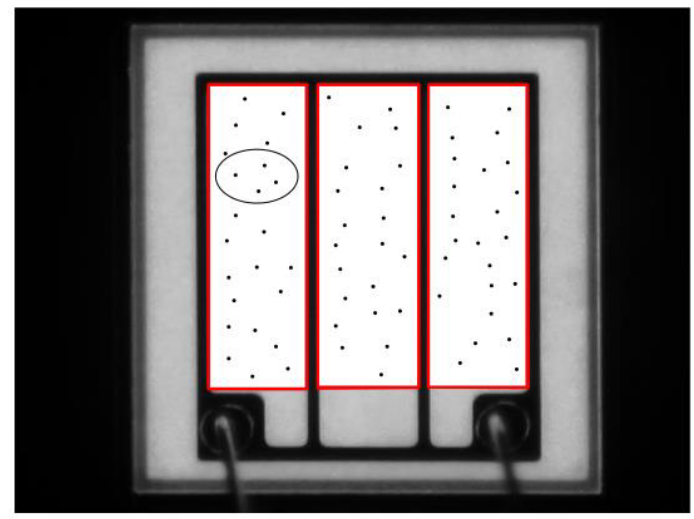

Figure 4. Sketch map of definition of dark points.

Image of the chip is segmented by image process technology referred before. Part of electrode is cut off and the uniform part that occupy the most part of the image in the surface of chip is got. Extracting the interested part from images of LED, like the part signed by red line in Figure 4 . Then the means of the whole pixel value in the segmented image is used to normalize it. Then a normalized part of segmented image can be obtained. The dark point in the image is defined as below:

In these segmented and normalized images, the pixel value less than a threshold which is set as 0.25 in this experiment is defined as dark point shown in Figure 4.

\subsection{The change of dark points with aging time increasing}

The images of aging for $300 \mathrm{~h}, 700 \mathrm{~h}, 1000 \mathrm{~h}$ and $1400 \mathrm{~h}$ is got. The interested part is extracted and the proportion of dark point that occupy the segmented part is calculated. A broken line of these data is draw as Figure5: 


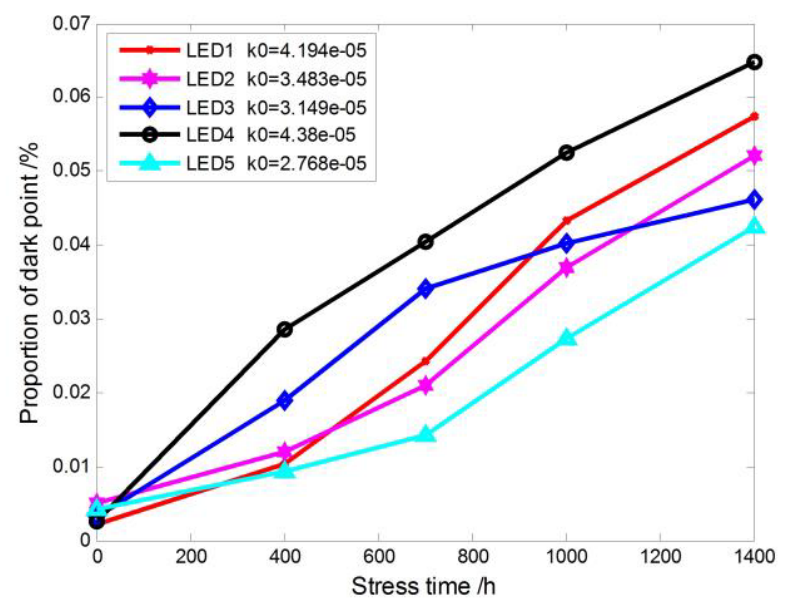

Figure 5. Proportion of dark points with stress time.

In Figure 5, the value of vertical coordinate is the proportion of dark points that occupy the segmented part of LED image. From the broken line graph, it is concluded that the density of the dark point is almost in direct proportion to aging time. The rate of the line is fitted out. For each curve, the slope to the horizontal axis, which is equal to the increasing rate of dark points, is defined as an index $\mathrm{k}_{0}$ shown in Figure 5. From the Figure 5, it can be concluded that each chip has its own $\mathrm{k}_{0}$, and $\mathrm{k}_{0}$ decides the increase rate of the dark points in the chip.

Based on the previous study [12-14], it is known that the darkening of the current-injection region is caused by the diffusion of point defects, and the density of the point defects have a relationship with the injected current. And the density of point defects was increased with the increase of non-radiative recombination centers in the active area. It is inferred that the dark area is caused by the increasing of non-radiative recombination centers. And from formulation (4) and the variation trend of Figure 4 , the relation between $\mathrm{D}_{\mathrm{c}}$ and $\mathrm{k}_{0}$ can be concluded:

$$
D_{c}=D_{0}+c k_{0} t
$$

In formulation (10), $\mathrm{k}_{0}$ is the increasing rate of the defect points referred before. And $\mathrm{c}$ is a constant coefficient, which can be got from Figure 4 by using the average of the five LEDs' initial value.

\subsection{Lifetime of LEDs with increasing rate of defect points}

As we known, the luminous flux satisfy the formulation below:

$$
p=p_{0} e^{-\beta t}
$$

In the formulation, the $\mathrm{p}_{0}$ is the initial value of luminous flux, $\beta$ is the attenuation coeffcient. Under rated operating current of LED, we measure the change of luminous flux with time variation, when $p=0.7 p_{0}$, the time is the lifetime of LED:

$$
\tau=\frac{-\operatorname{In} 0.7}{\beta}
$$

From formulation (9) and (10), the relation between $\mathrm{k}_{0}$ and the lifetime of LED is concluded as below:

$$
\tau=\frac{\frac{1}{L} \operatorname{In} \frac{a}{0.7}-D_{0}}{c k_{0}}
$$

As formulation (13) has only one variable parameter, then it can be simplified as below:

$$
\tau=\frac{m}{k_{0}}
$$

By measuring the luminous flux of these LEDs at $400 \mathrm{~h}, 700 \mathrm{~h}, 1000 \mathrm{~h}$ and $1400 \mathrm{~h}$, the lifetime of the LEDs can be calculated based on formulation (11). According to the lifetime of LEDs and $\mathrm{k}_{0}$, Figure 6 below is the fitted curve.

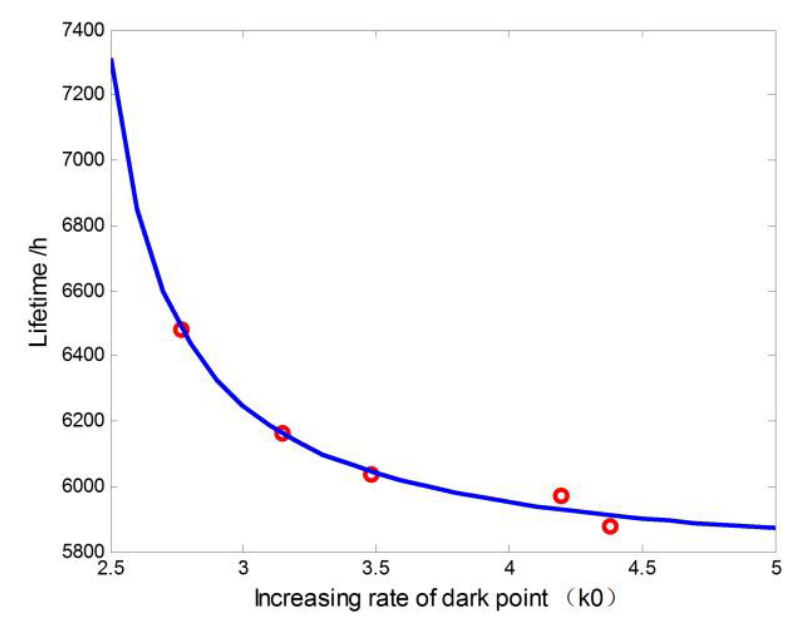

Figure 6. Proportion of dark points with stress time.

From this curve, it can be inferred the lifetime of a LED according to its diffusion rate of the defect points-$\mathrm{k}_{0}$. The lifetime of the same kind of LED can be estimated through the fitting formulation. And this method is not only effective but also simple to calculate the lifetime of LED.

\section{Conclusions}

A method of analyzing the reliability of high-power lightemitting diode (LED) by analyzing the chip image is proposed. This method can be used to all GaN-based LEDs. With the self-developed measuring system, chip image is taken and analyzed. The interested part of these images are extracted by using the algorithm of projection and the feature of LED's structure. An index called "dark point" to describe the reliability of LED is proposed. By counting up the variation trend of "dark point", it is found that the increasing rate of dark points is strongly associated with the reliability of LED as the LED with higher increasing rate has shorter lifetime. Through the relation between increasing rate of dark points and the 
lifetime of LED shown as formulation (14), the lifetime of LED can be calculated in a simpler way.

\section{References}

1. G. Zhang, Z. Chen, Phys, 33: 833-842(2004)

2. M. Meneghini, L. Trevisanell, G. Meneghesso, IEEE T Device Mat Re, 8:323-330(2008)

3. A. Hangleiter, F. Hitzel, C. Netzel, Phys. Rev. B, 95 : $1-4(2005)$

4. S.D. Lester, F.A. Ponce, M.G. Craford, Appl. Phys. Lett, 66: 1249-1251(1995)

5. X.A. Cao, P.M. Sandvik, S.F. LeBoeuf, Microelectron Reliab, 43: 1987-1991(2003)

6. X.A. Cao, K. Topol, F. Shahedipour-Sandvik, Proc. SPIE, 4776: 105-113(2002)

7. G. Meneghesso, S. Levada, R.Pierobon. Ieice T Electorn, E86-C: 2032-2038(2003)
8. G. Meneghesso, S. Levada, R. Pierobon, IEDM, 103106(2002)

9. G. Meneghesso, S. Levada, E. Zanoni, IRPS, 474478(2004)

10. F. Rossi, M. Pavesi, M. Meneghini, J. Appl. Phys, 99: 053104(2006)

11. T. Egawa, H. Ishikawa, T. Jimbo, Appl. Phys. Lett, 69, 830 (1996)

12. Tomiya, O. Goto, and M. Ikeda, Proc. IEEE 98(7), 1208-1213 (2010)

13. H. W. Shim, R. J. Choi, S. M. Jeong, L. Van Vinh, C.-H. Hong, E.-K. Suh, H. J. Lee, Y.-W. Kim, and Y. G. Hwang, Appl. Phys. Lett, 81:3552-3554(2002)

14. X. H. Wu, C. R. Elsass, A. Abare, M. Mack, S. Keller, P. M. Petroff, S. P. DenBaars, J. S. Speck, and S. J. Rosner, B Appl. Phys. Lett., 72:692694(1998). 